\title{
An overview of wireless sensor network and its applications
}

\author{
Khalid W. Al-Ani' ${ }^{1}$, Ahmed Subhi Abdalkafor ${ }^{2}$, Aiman Majid Nassar ${ }^{3}$ \\ ${ }^{1}$ Department of studies and planning, University Of Anbar, Iraq \\ ${ }^{2}$ Career Development Center, University Of Anbar, Iraq \\ ${ }^{3}$ College of Applied Sciences-Heet, University Of Anbar, Iraq
}

\begin{tabular}{|c|c|}
\hline Article Info & ABSTRACT \\
\hline Article history: & nall devices that can sense and collect data from the surrounding \\
\hline Received Jun 13, 2019 & $\begin{array}{l}\text { environment have been produced with the rapid development of wireless } \\
\text { technology and digital electronics. As a result, a wireless sensor network }\end{array}$ \\
\hline Revised Sep 15, 2019 & (WSN) was constructed from these sensor devices to send collected data to \\
\hline Accepted Sep 30, 2019 & $\begin{array}{l}\text { the base station for monitoring and analysis. This paper provides an overview } \\
\text { of WSN, as well as its architecture and applications. }\end{array}$ \\
\hline
\end{tabular}

Keywords:

Base station

Observer

Power saving

Sensor node

WSN

Copyright $@ 2020$ Institute of Advanced Engineering and Science. All rights reserved.

Corresponding Author:

Khalid W. Al-Ani,

Department of studies and planning,

University of Anbar, Anbar, Iraq.

Email: khaled_alani@uoanbar.edu.iq

\section{INTRODUCTION}

Wireless sensor network (WSN) consists of several small devices called 'nodes'. The nodes can sense, monitor and gather information from their surrounding environment. The collected information will be processed and sent to the base station through WSN for decision making. The WSN is a low-cost wireless network that can be rapidly deployed in hard-to-reach places, such as in an underground and underwater environment. The underground sensor nodes are provided with high transmission power to overcome the noisy channel attenuation, whereas the underwater sensor nodes are designed to be waterproof and resistant to moisture and salinity. However, this type of network is constrained by limited battery power, less bandwidth capacity, limited radio channel and processing capabilities. Therefore, the design of a WSN depends on the application and the environment to be monitored [1-2].

The WSN is used in diverse applications, such as health monitoring, process monitoring, disaster prevention and pipeline corrosion detection [3-4]. In recent years, WSN has received interest from researchers. Reference [5] presented a survey to address the challenges in WSN and to identify the issues that need to be considered in the implementation of WSN. The most prominent issues include energy saving, harsh environmental conditions, security and fault tolerance. Reference [6] presented another survey that discussed different security mechanisms to protect data from attackers. Reference [7] presented a literature survey on WSN and discussed the reduction of the gap between application and technology by unfolding the application issues. Reference [8] highlighted the application area for WSN, which was vast in spite of challenges caused by the power source and node mobility. Our paper presents an overview of WSN, focusing on its applications. The structure of our paper is presented as follows: Section 2 illustrates the structure of the sensor node, and Section 3 presents the operation of WSN. Section 4 provides the WSN communication protocol architecture. Section 5 focuses on the application WSN. Finally, Section 6 concludes the paper. 


\section{STRUCTURE OF SENSOR NODE}

A sensor node is a small and cheap device that forms a WSN. It consists of four essential units. The first unit corresponds to a sensing unit that is responsible for gathering data from its surrounding environment. The second unit refers to the processing unit, in which the gathered data will be processed. A processing unit is equipped with a memory to temporarily store the processed data. Subsequently, the processed data will be sent to another neighbor node or base station by the third unit called 'transceiver unit', which performs the transmitter and receiver operations to provide a communication wireless link to the sensor node. The fourth important unit is called the power supply unit, which is responsible for providing power to the sensor node. The advanced technology in power supply makes the use of solar energy possible to maintain battery power for long life power. Figure 1 shows the sensor node structure [9-10]. Figure 1 shows the structure of sensor nodes.

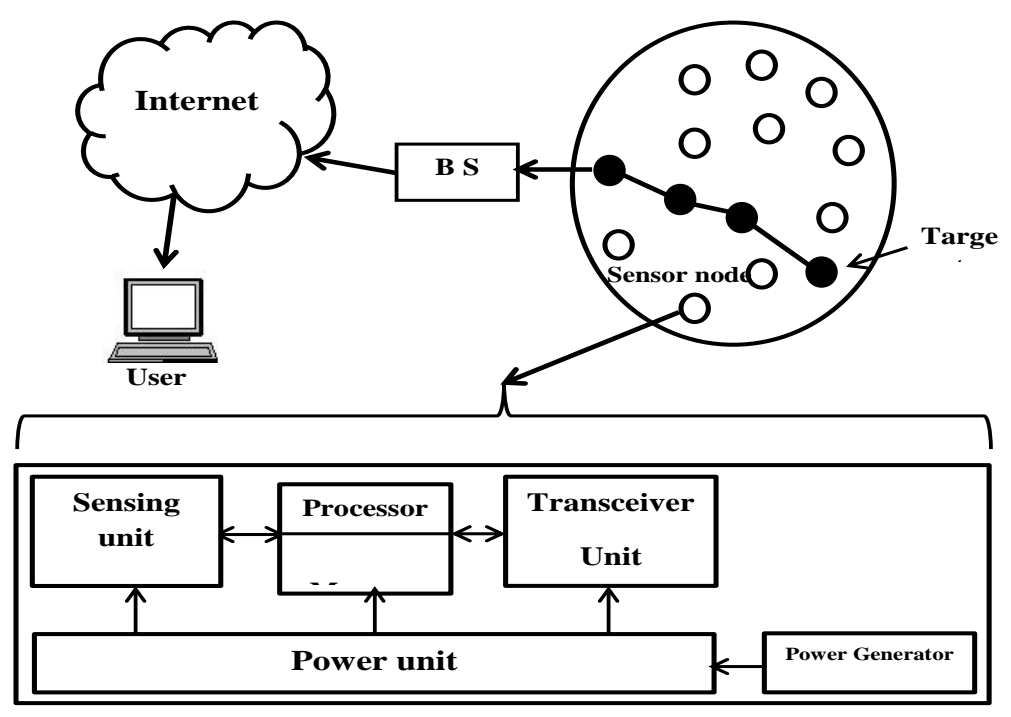

Figure 1. The structure of sensor nodes

\section{HOW WSN WORKS?}

The WSN structure consists of three components, namely, the sensor node, gateway and observer. Sensor nodes are responsible for sensing and gathering data from the surrounding environment. Subsequently, these nodes transmit data to the route node based on the routing protocol mechanism to avoid battery power limitations in the sensor nodes by adopting a multi-hop transmission technique. The route nodes perform the same function as the sensor node by sensing the environment and transmitting the collected data to the relay node. The relay node transmits the collected data directly to the base station (gateway). The gateway provides a connection to the network via the internet. Finally, the aggregated sensed data are transmitted to the observer (user) for monitoring and decision making in Figure 2 [11].

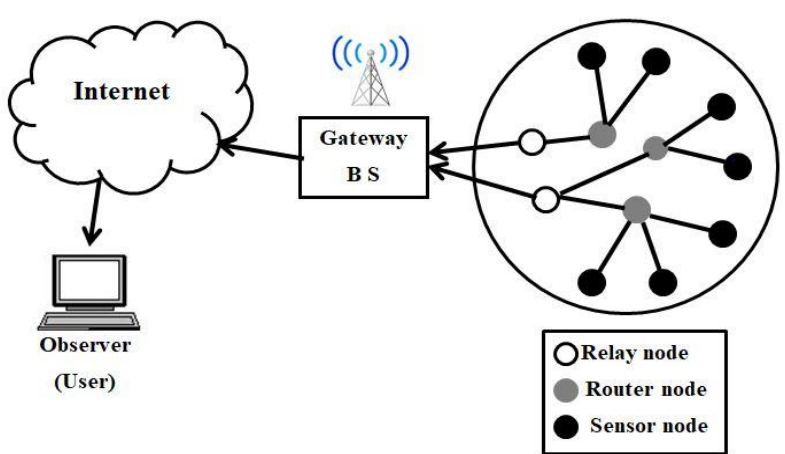

Figure 2. Structure of WSN 


\section{COMMUNICATION PROTOCOL ARCHITECTURE OF WSN}

The communication protocols of WSN must be well designed to preserve sensor network life and minimize energy consumption. These protocols consist of the following layers [12]:

\subsection{Physical Layer}

This layer preserves the lifetime of a sensor node by reducing energy consumption caused by selecting an optimal hop count and transmission power. It is also responsible for increasing the probability of transmission success by adopting a modulation process.

\subsection{Data Link Layer}

The data link layer is responsible for using a well-designed medium access control (MAC) protocol to establish a communication link amongst sensor nodes. MAC protocol must adapt to the topology change of a network caused by node failure and should preserve battery power.

\subsection{Network Layer}

This layer ensures that only the target nodes will consume battery power to receive data by using the sensor protocol for information via negotiation (SPIN). SPIN protocol prevents redundant data and minimizes power consumption.

\subsection{Transport Layer}

The transport layer is responsible for establishing a packet loss recovery mechanism, such as acknowledgment (ACK), to reduce transmission delay and energy consumption.

\subsection{Application Layer}

This layer is responsible for moving sensor nodes, switching sensor nodes on/off, monitoring data aggregation rules and reconstructing the sensor network basing on software operation provided by the sensor management protocol (SMP). Communication protocol architecture of WSN as shown in Figure 3.

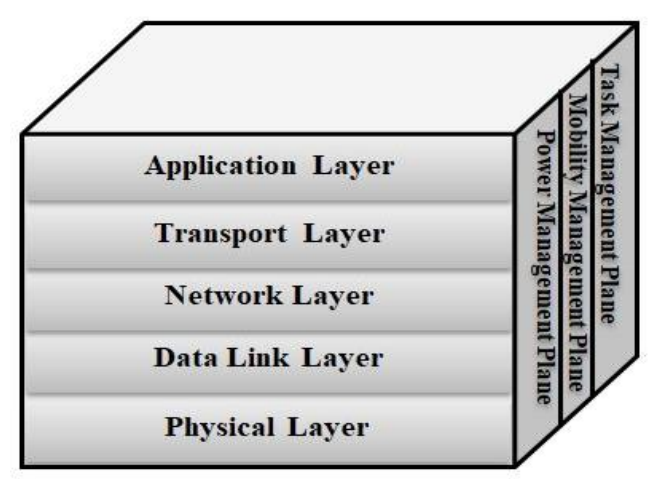

Figure 3. Communication protocol architecture of WSN

\section{WSN APPLICATIONS}

The WSN is used in a diverse range of application due to its characteristics, such as low cost and being rapidly deployed in any environment. It is adopted in a region and hardly accessed by observers underwater or underground. The following subsections describe WSN application.

\subsection{Healthcare Applications}

A WSN plays a prominent role in the healthcare domain by monitoring the vital signs of patients, including temperature, blood pressure and heartbeat [13]. The patient is equipped with a wireless medical sensor that it is wearable or implantable to transfer the information wirelessly to the base station for healthcare support. Reference [14] designed the wireless body sensor network to form a network and communicate with patients in the base station. This network also consists of four sensors that collects the physiological signals from the patient and relays the collected data to the base station. Furthermore, the data are analyzed by a specialist to provide proper health care treatment. Figure 4 shows the architecture of the health care system. 


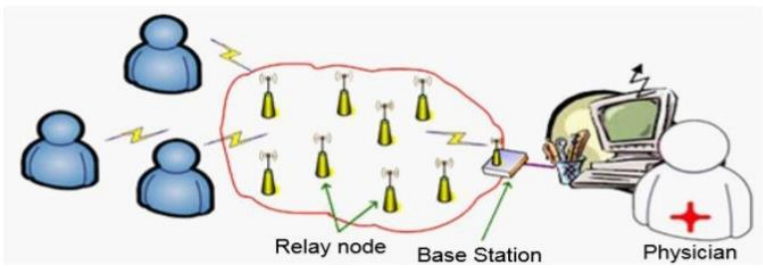

Figure 4. Architecture of the health care system in hospital

The WSN monitors and preserves the life of the elderly, especially those who are living independently. The acoustic sensor is used by WSN to detect the movement in the room and sent the monitored information to the control center. The caregiver will provide health care if something happens to the patient. Also, most of the elderly people are suffering from chronic illnesses. Therefore, remote healthcare is required to continue monitoring the patient's daily activities. WSN is suitable to be used for collecting the vital signs of the patient. Thus the patient is sent to the specialist for observation. Lastly, WSN is a suitable solution to provide a real-time monitoring of patients' health at their homes and to provide them with the appropriate health care to avoid traffic jams and waste of time in urban cities [15].

\subsection{Transportation Applications}

The rise of vehicles on the roads due to population growth requires an intelligent monitoring system to reduce traffic congestion, exhaust emissions and fuel consumption. WSN provides a transportation system to monitor the roads by fixing sensor nodes on roads and vehicles. The sensor nodes gather information, including traffic flow, speed and vehicle direction and weather condition before sending it to the base station for processing and analysis. The vehicles are equipped with portable laptops to observe information from the drivers to avoid traffic jam and accidents.

In public transportation, the arrival time to work is crucial for people who use public transportation. The WSN provides real-time information for the passengers at the bus station regarding the departure and arrival time, previous station location and bus location by fixing a sensor node for every bus to monitor its direction. WSN also ensures that the vegetables and fruits from rural arrive to urban areas in a short time by avoiding road congestion [16].

In addition, WSN provides intelligent parking management. Magnetic sensor nodes are installed on every parking slot to monitor the entrance and exit of a vehicle and send the parking information to the base station. A sensor node on a vehicle obtains the parking information from the base station and informs the driver about the available parking space and location [17]. Recognition is important, especially in terms of security, through the identification amongst images, such as car pictures and images of languages, databases, signatures and others [18-20]. Therefore, integrating image recognition techniques with the application of the image sensor node of the wireless network is possible in forming an integrated system that contributes to the integration of new wireless sensor networks, as well as identifying the images distributed within the network and distinguishes them from the rest of the images [21-25]. Transportation application of WSN as shown in Figure 5.

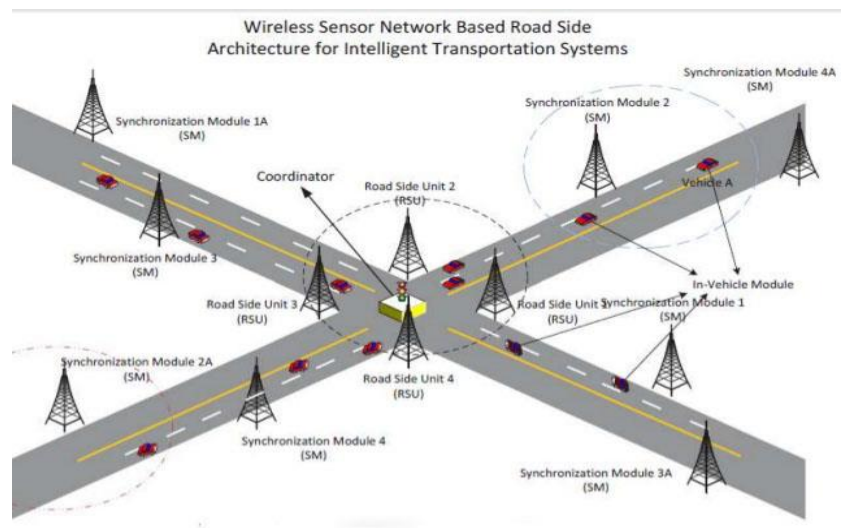

Figure 5. Transportation application of WSN 


\subsection{Industrial Applications}

WSN plays an important role in the industrial field by monitoring the manufacturing process at the production line, minimizing potential errors, predicting deterioration of the devices and scheduling algorithms for this application and others due to its significance for optimizing a real-time performance based on global information. [26-30]. In Pakistan, an industrial wireless sensor network has been utilized by [31] in the crockery industry unit. This industrial unit consists of two parts of dyes that are joined together by hydraulic pressure. The raw material poured on the lower dye and transformed from powder to solid after being heated, and the waste material stick out across the spillway. A pressure sensor is fixed on the spillway and sends signals to the control unit to prevent a bubble in the product by controlling the dyes open and close to kicking out the air between them. WSN reduces the financial loss and the need for experimental worker.

In structural monitoring, WSN is used to monitor the cracks in buildings to prevent collapse from taking place. WSN has also been established in Golden Gate Bridge in the USA to monitor the effects of wind and earthquakes on it by fixing multiple sensor nodes to gather the monitoring information and compare them with the ambient surroundings [32]. Furthermore, the water supply company has adopted WSN to monitor and control the water consumption by attaching a wireless vibration sensor to the pipes. The user can also observe the sensing information for rationalizing water consumption. The leaks in the pipelines can also be detected by WSN [32].

\subsection{Environment Applications}

WSN is used in a different type of environmental monitoring applications. The data gathered from its surrounding environment are observed to detect flood, earthquake, volcanic eruption and air pollution. For example, WSN was implemented on Great Duck Island to monitor the habits and activities of birds and activities during climate changes by distributing sensor nodes on the island. The aggregated data were sent to the base station through the gateway to analyze the living habits of the ducks. Monitoring the Volcan Revendator is another example for the application of WSN in environment application. The acoustic sensor nodes collected seismic data and sent it via radio link to a base station to monitor the volcanic activity in Figure 6 [33-34].

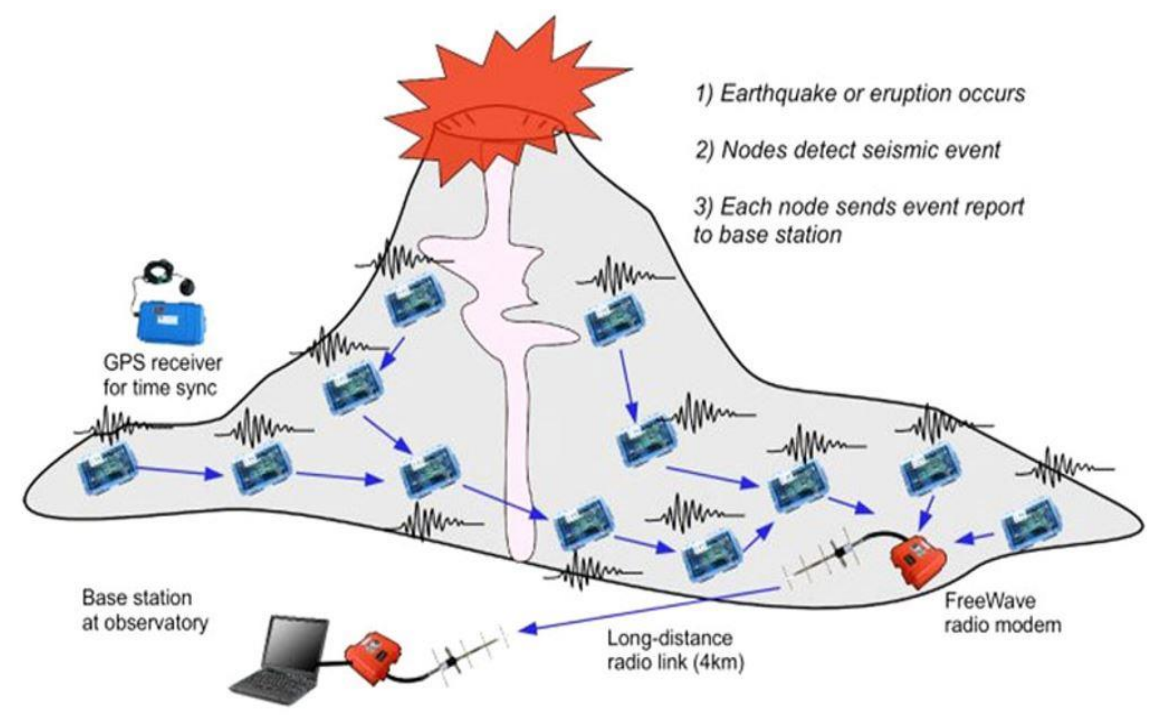

Figure 6. Volcano Revendator monitored by WSN

Air pollution monitoring has taken the biggest interest from most governments of the world due to the expansion of population and factories to provide a livable environment. Traditional networking is more expensive and restricted to a small geographic area. A WSN is established to monitor the air pollution of a long-distance area by using gas sensors that are equipped with radiofrequency. The gas sensors, such as $\mathrm{CO} 2$, $\mathrm{CO}$ and $\mathrm{NO} 2$, are measured and sent the collected information to the base station for observation and decision making [35]. 


\section{CONCLUSION}

WSN is a low-cost wireless network that can be rapidly deployed even in hard-to-reach places, such as underwater and underground environment. To understand the mechanism of this network, we presented the structure of the sensor node that is essential in the construction of WSN and its working and communication protocols. Furthermore, this paper reviewed the application of WSN in different domains using realistic examples that highlighted its importance.

\section{REFERENCES}

[1] O, Hicham, et al., "MG-leach: an enhanced leach protocol for wireless sensor network", International Journal of Electrical and Computer Engineering (IJECE), Vol. 9, No. 4, pp. 3139-3145, 2019.

[2] A. Gian Quoc,et al., "Flexible Configuration of Wireless Sensor Network for Monitoring of Rainfall-Induced Landslide", Indonesian Journal of Electrical Engineering and Computer Science, Vol. 12, No. 3, December 2018, pp. 1030-1036, 2018.

[3] Basavaraj G.N, Jaidhar C.D, "Low latency and energy efficient cluster based routing design for wireless sensor network," Indonesian Journal of Electrical Engineering and Computer Science (IJEECS), Vol. 13, No. 2, 2019.

[4] A. Davis and H. Chang, "A Survey of Wireless Sensor Network Architectures," International Journal of Computer Science and Engineering Survey, vol. 3, pp. 1-22, 2012.

[5] T. Bala, et al., "A Survey: Issues and Challenges in Wireless Sensor Networks", International Journal of Engineering and Technology, vol. 7, pp. 53-55, 2018.

[6] R. Sudha and B. Shamile, "A Survey on Wireless Sensor Networks," International Journal of Engineering Science and Research Technology, vol. 6, pp. 66-71, 2017.

[7] N. Singh and K. Rautela, " Literature Survey on Wireless Sensor Network," International Journal of Engineering and Computer Science, vol. 5. pp. 17544-17548, 2016.

[8] K.T. Kishor, et al., "A Fundamental Implementations and Working Principles of Wireless Sensor Networks," Compusoft, vol. 4, pp. 2030-2035, 2015.

[9] K. N., Jyoti Metan, K. N. Narashinha Murthy, "N-tier modelling of robust key management for secure data aggregation in wireless sensor network", International Journal of Electrical and Computer Engineering (IJECE), Vol. 9, No. 4, pp. 2682-2690, 2019.

[10] A. Basim, A. Yasser Kareem, "Lifetime enhancement for clustering protocols in heterogeneous wireless sensor networks", Indonesian Journal of Electrical Engineering and Computer Science (IJEECS), Vol. 14, No. 3, pp. 1305-1314, 2019.

[11] J, Youngjun, et a, l" Design and implementation of heterogeneous surface gateway for underwater acoustic sensor network", International Journal of Electrical and Computer Engineering (IJECE), Vol. 9, No. 2, pp. 1226-1231, 2019.

[12] H.M.A. Fahmy, "Protocol Stack of WSNs. "Wireless Sensor Networks", Springer, Singapore, 2016. 55-68.

[13] R. K., Saini, R., Ritika, \& S. Vijay, "Data flow in wireless sensor network protocol stack by using Bellman-Ford routing algorithm". Bulletin of Electrical Engineering and Informatics (BEEI), 6(1), 81-87, 2017.

[14] M. Aminian and H.R. Naji., "A Hospital Healthcare Monitoring System Using Wireless Sensor Networks," $J$. Health Med. Inform, vol. 4, pp. 121, 2013.

[15] A. Minaie, et al., "Application of Wireless Sensor Networks in Health Care System," 120th ASEE Annual Confrerence and Exposition, age 23.1, 2013.

[16] X. Hu, et al., "Application Scenarios of Wireless Sensor Networks for Urban Transportation: A Survey," 34th Chinese Control Conference (CCC), IEEE, 2015.

[17] M.A. Kafi, et al., "A Study of Wireless Sensor Networks for Urban Traffic Monitoring: Applications and Architectures," Procedia Computer Science, vol. 19, pp. 617-626, 2013.

[18] A.S., Abdalkafor,"DFRS-database for fingerprint recognition system using Ink-On-Paper technique, Journal of Engineering and Applied Sciences, Vol 13, 7, pp. 7401-7407, 2018.

[19] A. S. Abdalkafor, et al., " A Novel Database for Arabic Handwritten Recognition (NDAHR) System”, In 2019 2st International Conference on Computer Applications \& Information Security (ICCAIS), 2019.

[20] Abdalkafor, A. S., Awad W. K, Alheeti, K. M. A. "Novel Comprehensive Database For Arabic And English OffLine Handwritten Digits Recognition", Journal Of Southwest Jiaotong University, in press, 2019.

[21] Z. Y, Cao,Z.Z. Ji, \&, M. Z.Hu, (2005, April). "An image sensor node for wireless sensor networks". In International Conference on Information Technology: Coding and Computing (ITCC'05), Vol. 2, pp. 740-74, 2005.

[22] A.S. Abdalkafor "Survey for Databases on Arabic Off-line Handwritten Characters Recognition System". In 2018 1st International Conference on Computer Applications \& Information Security (ICCAIS), IEEE, pp. 1-6, 2018.

[23] A. S. Abdalkafor, "Designing Offline Arabic Handwritten Isolated Character Recognition System using Artificial Neural Network Approach". International Journal of Technology, 8(3), 528-538, 2017.

[24] A. S. Abdalkafor \& Sadeq, A, "Arabic Offline Handwritten Isolated Character Recognition System Using Neural Network". International Journal of Business and ICT, 2(3), 41-50, 2016.

[25] D, Duran, et al, "Hierarchical character oriented wildlife species recognition through heterogeneous wireless sensor networks". In 2007 IEEE 18th International Symposium on Personal, Indoor and Mobile Radio Communications, pp. 1-5, 2007.

[26] A. A, Jihad \& A.S, Abdalkafor, "A Framework for Sentiment Analysis in Arabic Text", Indonesian Journal of Electrical Engineering and Computer Science (IJEECS), Vol 16, No 3, 2019. 
[27] A. S. Abdalkafor, Aiman, M.N \& Mustafa, N.O, "Predicting The Success Rates of Schools Using Artificial Neural Network". Journal of Theoretical and Applied Information Technology, no. 96, pp. 6339-6348, 2018.

[28] X. Jin, A. Saifullah, C. Lu, \& P. Zeng, "Real-Time Scheduling for Event-Triggered and Time-Triggered Flows in Industrial Wireless Sensor-Actuator Networks". In IEEE INFOCOM 2019-IEEE Conference on Computer Communications, pp. 1684-1692, 2019.

[29] A.S., Abdalkafor, H.M, Taher, H. M., \& K. W. Al-Ani, "A Novel Method Based On Priority For Enhancement Round-Robin Scheduling Algorithm". Journal of Theoretical \& Applied Information, Technology, 96(13) P. 4092-4102, 2018.

[30] O. A. Mahdi, et al, "An Energy-Aware and Load-balancing Routing scheme for Wireless Sensor Networks." Indonesian Journal of Electrical Engineering and Computer Science (IJEECS), Vol. 12, No. 3, PP. 1312-1319, 2019.

[31] F. Zafar, et al., "Application of WSN in Crockery Manufacturing Industry." IJASOS-International E-journal of Advances in Social Sciences, vol. 2, pp. 461-470, 2016.

[32] M. Erdelj, et al., "Applications of Industrial Wireless Sensor Networks," Industrial Wireless Sensor Networks: Applications, Protocols, and Standards, pp. 1-22, 2013.

[33] S.R. Boselin, et al.,"Wireless Sensor Network Based Smart Environment Applications," International Journal of Innovative Research in Technology, 2017.

[34] A, Raja Waseem, Z, AnazidaI, Saleem"Systematic literature review on designing trust-based security for WSNs", Indonesian Journal of Electrical Engineering and Computer Science (IJEECS), Vol. 14, No. 3, June 2019, pp. 1395-1404, 2019.

[35] B. Bathiya, et al., "Air Pollution Monitoring Using Wireless Sensor Network," IEEE International WIE Conference on Electrical and Computer Engineering (WIECON-ECE), IEEE, 2016.

\section{BIOGRAPHIES OF AUTHORS}

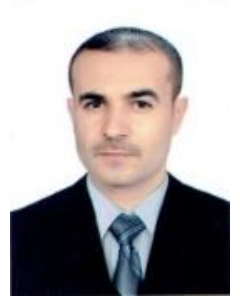

Khalid W. Al-Ani was born in Al-Anbar Govrnorate, Iraq in 1982. He received the B.Sc. Computer Engineering and Information Technology degree from the University of Technology, Iraq in 2005 and the Master degree in Information Technology from University Tenaga Nasional (UNITEN), Malaysia in 2014. He is currently one of the teaching staff at the University of Anbar, Iraq. His research interests include data communication and networks, big data, data base, and artificial intelligence.

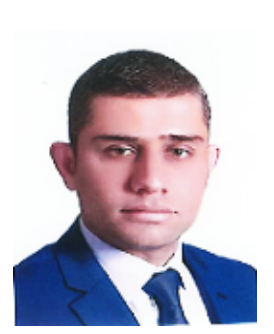

Ahmed Subhi Abdalkafor was born in Anbar-Iraq in 1988. He received his B.Sc. from Faculty of Computer Science at Anbar University, Iraq in 2010. The MSc. degree from Computer Science Department in Middle East University, Jordan in 2016. His research interests are, Image Processing, Pattern Classification, Artificial Intelligent, Neural Network, Cloud Computing, Machine Learning and Data Mining.

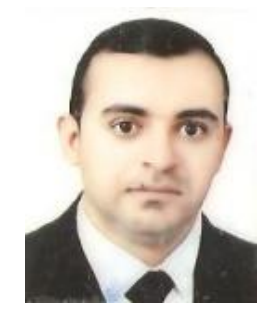

Aiman Majid Nassar was born in Anbar-Iraq in 1987. He received his B.Sc. from Faculty of College of Education for Pure Sciences at Anbar University, Iraq in 2010. The MSc. degree from Mathematics Science Department in The Sam Institute, India in 2013. His research interests are, a study of ordinary differential equation throgh numerical analysis. I am currently teaching at the Faculty of Applied Sciences, Anbar University, Iraq 